Physics Vol. 3, No. 4, pp. 199-219, 1967. Physics Publishing Co. Printed in Great Britain.

\title{
ELECTROSTATIC EFFECT AND SUPERCONDUCTIVITY IN TINY SAMPLES*
}

\author{
DAVID MARKOWITZ $\dagger$ \\ Physics Department and Institute of Materials Science \\ The University of Connecticut, Storrs, Connecticut \\ (Received 12 September 1966)
}

\begin{abstract}
It is our notion that the Anderson phase-number relationships (which he developed for the Josephson effect) should be applied to a superconductor in equilibrium. This adds to the BCS postulates a further one: there exists an intrinsic phase spread across a suitable domain in a sample and a corresponding uncertainty in the number of electrons in that domain. The new Hamiltonian contains a Coulomb repulsion term, and the new wave function contains unpaired occupied states even in the ground state. Minimization of the energy shows that the size of the domain within which the phase spread exists is the size of the sample. Detailed numerical solution finds that only for beads of 100-500 $\AA$ will the results differ significantly from the BCS or bulk case. Excited states and thermal properties are solved for in detail and other properties are discussed. Predictions are a smaller gap and a lower critical temperature than in bulk, a first order transition to the normal state, and an effective density of states for single particle transitions at very low temperatures which exceeds that in the normal state. It is well known that the samples in Knight shift experiments are of the size necessary to test these predictions; however, the effects predicted here are not found. We argue that those samples are not in electrical isolation, in violation of a condition critical for the detection of the electrostatic effect.
\end{abstract}

\section{Introduction}

JOSEPHSON [1] showed that condensed pairs can tunnel from one.superconductor to another with zero voltage drop. Anderson [2] put the Josephson effect on an intuitively satisfying basis through his analysis of phase and particle number relationships.

It is our notion that these relationships, so successfully applied to a nonequilibrium

- Work supported by the U.S. Air Force office of Scientific Research Grant AF-AFOSR-474-64.

t The author began this work while holding a research associateship at Rutgers, The State University, New Brunswick, New Jersey. He was supported by National Science Foundation Grant NSF - G 24277. 
problem, should be invoked for equilibrium situations. Anderson, in his original paper, in effect suggested such an extension when he wrote down a wave function which fixed the number of particles at the expense of an indeterminate phase. In this paper the superconducting ground state and then the equilibrium state at $T>0$ will be examined from this point of view. We shall not change any of the fundamental BCS [3] postulates, such as the introduction of the truncated Hamiltonian, the pairing scheme, and the Hartree assumption of independent probabilities for individual state occupation. The main thing we do is to include an additional postulate, namely that there exists an intrinsic spread in phase across a domain in a superconducting sample and, linked to this via the uncertainty principle, a pair number density uncertainty.

The origin of this effect may be explained in a manner which draws on Anderson's discussion. Think of two neighboring regions in a superconductor. To have the maximum correlation energy, they would prefer to have the same phase. However, this would produce an infinite uncertainty in the relative electron number, which implies huge charge densities for a substantial fraction of the configurations specifying the state. The superconductor would prefer to have a uniform charge density. Since it cannot have both that and a constant phase, it optimizes the two. The same argument holds for any such imaginary division of the sample. We emphasize that this behavior of the phase is different from, and also independent of, the standard phase uncertainty which refers to our ignorance of a phase which is supposed to be unvarying over the sample.

These effects share the features of all zero-point fluctuations. Anderson [4] has shown that the occurrence of this kind of behavior is widespread in systems governed by a Hamiltonian which possesses a symmetry not possessed by the wave function. This symmetry-breaking wave function arises when the system undergoes a phase transformation to an ordered state. In the case of the superconductor, the order parameter is a complex number, $\Delta e^{i \phi}$, and states with different values of phase $\phi$ are degenerate. There results the zero-point fluctuations in phase. 0ther systems exhibiting this kind of phenomenon are better known and include lattice structures and magnetically ordered systems.

\section{The Model}

The BCS Hamiltonian will be amended by adding a term representing the electrostatic repulsion of an excess (or deficiency) of electrons, $H_{e}$. The revised Hamiltonian, appropriate to unit volume, is

$$
H=H_{K E}+H_{I}+H_{e}
$$

where

$$
\begin{aligned}
& H_{K E}=\sum_{k>k_{f}, s} \varepsilon_{k} n_{k s}+\sum_{k<k_{f}, s}\left|\varepsilon_{k}\right|\left(1-n_{k s}\right), \\
& H_{I}=-\sum_{k, k}, V_{k k^{\prime} b_{k}^{+} \cdot b_{k},} \\
& H_{e}=\frac{1}{r^{3}} c \frac{e^{2}}{r}<\left[\sum_{k, s}\left(n_{k s}-<n_{k s}>\right)\right]^{2}>.
\end{aligned}
$$


As in BCS, $n_{k s}$ is the number operator, $b_{k}^{+}$the pair creation operator, $\varepsilon_{k}$ the kinetic energy measured from the Fermi level. In $H_{e}$ the angular brackets denote an expectation value. We are allowing the sample an extra degree of freedom: namely, that it may divide into domains of volume $r^{3}$ within each of which the same phase spread and electron excess exist. The constant $c$ is of order one and depends on the shape of the charge distribution.

A suitable wave function to be used as a variational approximation to the exact ground state will be formed from a product function of pair states and singly occupied states with occupancy probability functions $h_{k}$ and $l_{k}$, respectively [5]. The reason for believing that singles exist in the ground state is that electrostatic energies are enormous and intolerable to the system. Consider, for example, an excess of one pair inside a region with $r=5 \times 10^{-5} \mathrm{~cm}$, a typical coherence distance. The repulsive energy is 10-2 V or ten times a typical gap energy. It is energetically favorable to have two single particles (or holes) above the gap neutralize the excess charge.

The approximate ground state wave function for each domain $r^{3}$ is

$$
\begin{aligned}
& \Psi_{0}(\phi)=\int_{0}^{2 \pi} d \phi^{\prime} F\left(\phi-\phi^{\prime}\right) e^{i n \phi^{\prime}} \\
& \times{ }_{k}^{\pi}\left\{\left(1-l_{k}\right)^{1 / 2}\left[\left(1-h_{k}\right)^{1 / 2}+e^{i \varphi^{\prime}} h_{k}^{1 / 2} b_{k}^{+}\right]\right. \\
& \left.+e^{i \Phi_{k} l_{k}^{1 / 2} a_{k}^{+}}\right\} \mid 0>.
\end{aligned}
$$

Each domain has its phase centered about the same value $\phi$. (One could at the end set the complete wave function $\Psi_{0}$, for an isolated system, equal to $(2 \pi)-1 \int_{0}^{2 \pi} \Psi_{0}\left(\varphi_{)} d \varphi_{0}\right)$. Since $\varphi_{k}$ is arbitrary, the singles are not restricted by the uncertainty principle. In the absence of a theory of phase fluctuations, a worthwhile thing to do is to examine the consequences of assuming a reasonable form for $F\left(\phi-\phi^{\prime}\right)$. The form we choose is

$$
F\left(\phi-\phi^{\prime}\right)=a \exp \left[-\left(\phi-\phi^{\prime}\right)^{2} / \sigma^{2}\right] \text {. }
$$

The reason for this choice is that a Gaussian yields the minimum uncertainty product. The quantity $\sigma$ is the root mean square deviation in $\phi$. In writing $\Psi_{0}(\varphi)$ as a product function, separable in $\sigma$ and the $h_{k}$, we have extended the Hartree assumption to include $\sigma$. Because of the presence of singly-occupied states (i.e. an apparent "normal" component) in the superconducting ground state, and because the names "intermediate state" and "mixed state" have been preempted, we have termed our imodel "the motley state".

If $\Psi_{0}$ is used to evaluate the expectation value of $H$, we find for the ground state energy [6]

$$
W_{0}=\sum_{k}\left|\varepsilon_{k}\right|\left[l_{k}+2\left(1-l_{k}\right) h_{k}\left(\left|\varepsilon_{k}\right|\right)\right]
$$




$$
\begin{aligned}
& -\cos \sigma \sum_{k, k^{\prime}} V_{k k^{\prime}}\left[h_{k}\left(1-h_{k}\right) h_{k} \cdot\left(1-h_{k^{\prime}}\right)\right]^{1 / 2}\left(1-l_{k}\right)\left(1-l_{k^{\prime}}\right) \\
& +\frac{4 c e^{2}}{r^{4}}\left(\frac{1}{\sigma}-\frac{1}{2} r^{3} \sum_{k} l_{k}\right)^{2} .
\end{aligned}
$$

The kinetic and pairing energy terms are in a form analogous to equation (3.16) of BCS, which is their finite temperature form. The last term is obtained by taking the uncertainty principle literally: that is, $n_{1} \sigma=1$, where $n_{1}$ is the excess number of pairs.

\section{The Ground State}

To find the ground state, we minimize $W_{0}$ with respect to $h_{k}, l_{k}, \sigma$, and $r$. We find, with respect to $h_{k}$,

$\frac{\partial W_{0}}{\partial h_{k}}=\left(1-l_{k}\right)\left\{2 \varepsilon_{k}-\frac{1-2 h_{k}}{\left[h_{k}\left(1-h_{k}\right)\right]^{1 / 2}} \cos \sigma \sum_{k, k^{\prime}} V_{k k^{\prime}}\left[h_{k} \cdot\left(1-h_{k}^{\prime}\right]^{1 / z}\left(1-l_{k}^{\prime}\right)\right\}\right.$

Following BCS, we assume $V_{k k^{\prime}}=V$ with a cutoff at $\omega$, the Debye frequency. Setting (6) equal to zero, we conclude that either $l_{k}=1$ or else the BCS-like set of relations must be obeyed:

$$
\begin{gathered}
h_{k}=\frac{1}{2}\left(1-\varepsilon_{k} / E_{k}\right),\left[h_{k}\left(1-h_{k}\right)\right]^{1 / 2}=\frac{1}{2} \Delta / E_{k}, \quad E_{k}^{2}=\varepsilon_{k}^{2}+\Delta^{2}, \\
\Delta=V \cos \sigma \sum_{k^{\prime}}\left[h_{k} \cdot\left(1-h_{k^{\prime}}\right)\right]^{1 / 2}\left(1-l_{k^{\prime}}\right) .
\end{gathered}
$$

We also find

$$
\begin{aligned}
\frac{\partial W_{0}}{\partial l_{k}}=\varepsilon_{k}\left(1-2 h_{k}\right) & +2 \cos \sigma \sum_{k^{\prime}} V_{k k^{\prime}}\left[h_{k}{ }^{\prime}\left(1-h_{k^{\prime}}\right]^{1 / 2}\left(1-l_{k}{ }^{\prime}\right)\right. \\
& \times\left[h_{k}\left(1-h_{k}\right)\right]^{1 / 2}-\frac{4 c e^{2}}{r}\left(\frac{1}{\sigma}-\frac{1}{2} r^{3} \sum_{k^{\prime}} l_{k^{\prime}}\right),
\end{aligned}
$$

and, using the relations (7) and (8) plus the definition

$$
\sum_{k} l_{k}{ }^{\prime}=2 N \varepsilon_{1}
$$

( $N$ is the normal density of states of one spin at the Fermi surface), 


$$
\frac{\partial W_{0}}{\partial l_{k}}=E_{k}-\frac{4 c e^{2}}{r}\left(\frac{1}{\sigma}-r^{3} N \varepsilon_{1}\right) \equiv E_{k}-E_{2}
$$

In a moment we will see the physical meaning of $E_{2}$. For now, assuming that $E_{2}$ will have a specific value, we see that

$$
\partial W_{0} / \partial l_{k}<0(>0) \text { for } E_{k}<E_{2}\left(>E_{2}\right) \text {. }
$$

This means that $l_{k}$ is as large (small) as possible for $E_{k}<E_{2}\left(>E_{2}\right)$. But $l_{k}$ cannot be larger than definite single occupation nor smaller than zero. Hence $l_{k}=1$ for $E_{k}<E_{2}$ and $l_{k}=0$ for $E_{k}>E_{2}$; also $l(-\varepsilon)=l(\varepsilon)$. These values for $l_{k}$ produce $\Sigma_{k} l_{k}=2 N \varepsilon_{2}$, and thus $\varepsilon_{2}=\varepsilon_{1}$ and $E_{2}=E_{1}$, where

$$
E_{1}^{2}=\varepsilon_{1}^{2}+\Delta^{2}
$$

Setting $\partial W_{0} / \partial l_{k}=0$ yields

$$
E_{1}=\frac{4 c e^{2}}{r}\left(\frac{1}{\sigma}-r^{3} N \varepsilon_{1}\right) .
$$

It is intuitively gratifying that the singles occupy the lowest available states above the gap. The term "gap" in this context means the energy region containing no allowed states. The minimum energy for scattering a single is zero. The minimum energy for breaking a pair is $2 E_{1}$.

We minimize $W_{0}$ with respect to $\sigma$ and use equation (12) in the resulting equation to obtain

$$
A(\sigma)=\sigma^{2} \tan \sigma \sec \sigma=\frac{2 V E_{1}}{r^{3} \Delta^{2}} .
$$

A second expression relating $\sigma, \Delta$, and $\varepsilon_{1}$ comes from equation (8), which may be solved in the form

$$
(N V \cos \sigma)^{-1}=\int_{\varepsilon_{1}}^{\omega} \frac{d \varepsilon}{E}=\ln \frac{\omega+\Omega}{\varepsilon_{1}+E_{1}} .
$$

where $\Omega^{2}=\omega^{2}+\Delta^{2}$. The corresponding BCS formula is

$$
(N V)-1=\int_{0}^{\omega} \frac{d \varepsilon}{E_{b}}=\ln \frac{\omega+\Omega_{b}}{\Delta_{b}} .
$$

where the subscript $b$ refers to the values of the parameters in the BCS limit, which we shall show applies to bulk material. We now appeal to the weak-coupling approximation, which says that $\Delta \ll \omega$ and so $\Omega \approx \Omega_{b}$. Combining (14) and (15) then gives

$$
E_{1}+\varepsilon_{1}=\Delta_{b} \exp \left[-\frac{\sec \sigma-1}{N V}\right]=B(\sigma)
$$


For values of $r$ smaller than a coherence distance, we have seen in the paragraph above equation (3) that $\Delta \ll 4 c e^{2 / r}$. Therefore, a final needed expression comes from (12) in the simplified form

$$
\varepsilon_{1}^{-1}=r^{3} N \text { o. }
$$

We shall later show that this equation is valid for any value of $r$.

When we attempt to minimize $W_{0}$ with respect to $r$, we find that $\partial W_{0} / \partial r$ is always negative, implying that $W_{0}$ is a minimum for $r \rightarrow \infty$. In this limit, $\sigma \rightarrow 0, \varepsilon_{1} \rightarrow 0, \Delta \rightarrow \Delta_{b}$. This is the BCS or bulk limit. Thus in this limit the electrostatic effect per unit volume goes to zero.

It is apparent that the superconductor will choose $r$ to be the size of the sample, and so we ask how small must $r$ be for the sample not to be "bulk", and what is the behavior of the motley state as we decrease $r$ toward the limit of the smallest possible sample that is still superconducting?

\section{Numerical Solution}

We need to solve, simultaneously, for $\sigma, \Delta$, and $\varepsilon_{1}$, in the system of equations, (11), (13), (16 and (17). For this purpose we assume values for the bulk parameters which will be representative of real metals such as $\mathrm{Sn}$, In, $\mathrm{Hg}, \mathrm{Pb}$; and others: namely, $N V=1 / 3,2 \mathrm{~V}=10^{-22} \mathrm{~V} \mathrm{~cm}^{3}$, $\Delta_{b}=10^{-3} \mathrm{~V}$. Our plan is simple to state but tedious to carry out. We tabulate the functions $A(\sigma)$ and $B(\sigma)$ appearing in equations (13) and (16) for values of $\sigma$ between 0.1 and 0.8 . For any particular choice of $r$, we at first neglect $\varepsilon_{1}$, so that (16) becomes $\Delta=B(\sigma)$ and (13) becomes $\Delta^{-1}=\left(r^{3} / 2 h\right) A(\sigma)$. We search our table of $A(\sigma)$ and $B(\sigma)$ for the values that give the same $\Delta$. We then use $\varepsilon_{1}$ to improve upon these values, iteratively.

In Fig. 1 we have plotted the results for $\sigma$, for $\Delta \Delta_{b}$, and for $\varepsilon_{1} / \Delta$ vs. $r$ in a range of $r$ around $100-300 \check{A}$. Two special values of $r$, called $r_{0}$ and $r_{s}$, are defined later in this section For $r>300 \AA$. (13) becomes $\sigma r=\left(2 V / \Delta_{b}\right)^{1 / 3}=4.6 \times 10^{-7} \mathrm{~cm}$ and (16) becomes $\Delta=\Delta_{b}\left(1-1.5 \sigma^{2}\right)$, both to an accuracy of a few per cent. On this basis, a bead with $r>300 \AA$ is practically "bulk" material. For $r<r_{s}$ where $r_{s}$ is estimated to be $\widetilde{>} 90 \AA$, there is no solution to our equations and the motley state does not exist. Using the large $r$ form for $\sigma$ in (12), we find. that $\sigma^{-1} \gg E_{1} /\left(4 c e^{2} / r\right)$ for all $r$ and, therefore, $\sigma^{-1}=r^{3} N \varepsilon_{1}$ for all $r$, as we asserted earlier.

Even for $r>r_{s}$, the result that a motley state exists is not a guarantee that it is stable relative to the normal state. Indeed, we expect that when $\varepsilon_{1} \sim \Delta$, the energy of the singles above the gap outweighs the pairing energy. We determine $W_{0}$ by casting equation (5) into the form

$$
W_{0}=2 N \int_{0}^{\varepsilon_{1}} \varepsilon l(\varepsilon) d \varepsilon+4 N \int_{\varepsilon_{1}}^{\omega} \varepsilon h(\varepsilon) d \varepsilon-\frac{\Delta^{2}}{V \cos \sigma}+\frac{E_{1}{ }^{2}}{\left(4 c e^{2} / r\right) r^{3}} .
$$

This expression is analogous to equation (2.41) of BCS. We have used equations (8) and (12). In the weak-coupling limit we find 


$$
\frac{W_{0}}{W_{b}}=\frac{\Delta^{2}-2 \varepsilon_{1} E_{1}}{\Delta_{b}{ }^{2}}-\frac{2 E_{1}{ }^{2} / \Delta_{b}{ }^{2}}{\left(4 c e^{2} / r\right) r^{3}} .
$$

in terms of the bulk or BCS energy $w_{b}$;

$$
W_{b}=-\frac{1}{2} N \Delta_{b}^{2} \text {. }
$$

The denominator of the last term in (19) is far greater than one, implying that that term is negligible. In particular, for large $r$, while the electrostatic energy of the sample is large, that energy per unit volume is very small and inaccessable to experiment.

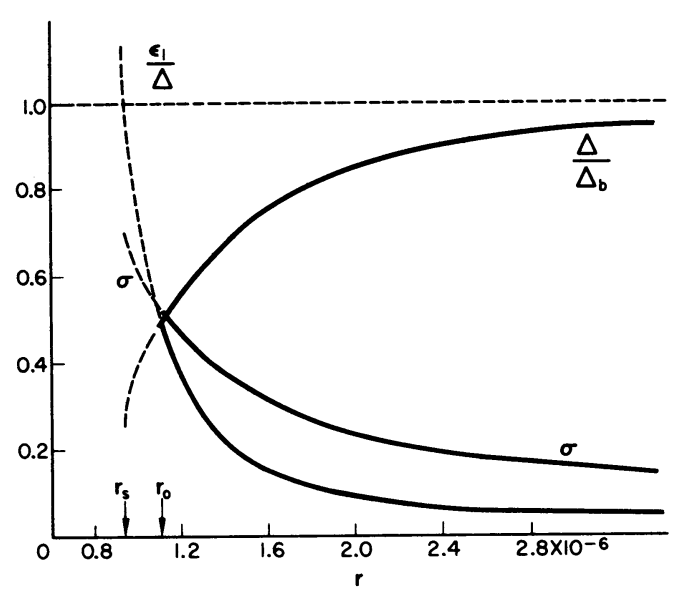

FIGURE 1

\begin{abstract}
The motley parameters, $\sigma, N / \Delta_{b}$ and $\varepsilon_{1} / \Delta$, vs. $r$ at $T=0 . \sigma$ is the intrinsic phase spread, $\Delta$ is the energy gap, $\Delta_{b}$ is the bulk energy gap, $\varepsilon_{1}$ is the highest Bloch energy occupied by a single, and $r$ is the dimension of the sample. The dashed portions denote the region of metastability. $r_{s}$ is the smallest value of $r$ such that a solution exists, while $r_{0}$ is the smallest value of $r$ such that the solution is stable with respect to the normal state.
\end{abstract}

In Fig. 2 we have plotted the results for $W_{0} / W_{b}$ vs. $r$. Apparently we have to go out to 500 or $600 \AA$ in order to have $W_{0}=W_{b}$ to a few per cent. On this basis, $r>600 \AA$ is essentially "bulk" material. At a value $r_{0} \approx 110 \AA, W_{0}=0$ relative to the normal state. The region below $r_{0}$ is dashed on Figs. 1 and 2. Note that $\varepsilon_{1}\left(r_{0}\right)=0.55 \Delta\left(r_{0}\right)$. The meaning of negative $W_{0}$ is, of course, that the motley state is metastable.

It is plausible that the motley state should exhibit its most marked behavior for values of $r \sim 10^{-6} \mathrm{~cm}$. The two characteristic lengths in the problem are $\xi$, which is the correlation distance, usually $\sim 10^{-4}$, and $d \sim\left(N \varepsilon_{F}\right)^{-1 / 3}$, the interparticle spacing, which is $\sim 10^{-8}$ and is determined by a balance of electrostatic and kinetic energies. Since the motley state is itself a compromise between electrostatic and correlation energies, we expect a dominant role to be 
played by distances given by some relation like $r \sim(\xi d)^{1 / 2} \sim 10^{-6} \mathrm{~cm}$.

We remind the reader that an approximation [6] leading to equation (5) worsens as $\sigma$ increase Thus it is likely that our values for $r_{8}$ and $r_{0}$ are somewhat incorrect. The qualitative feature should not be affected

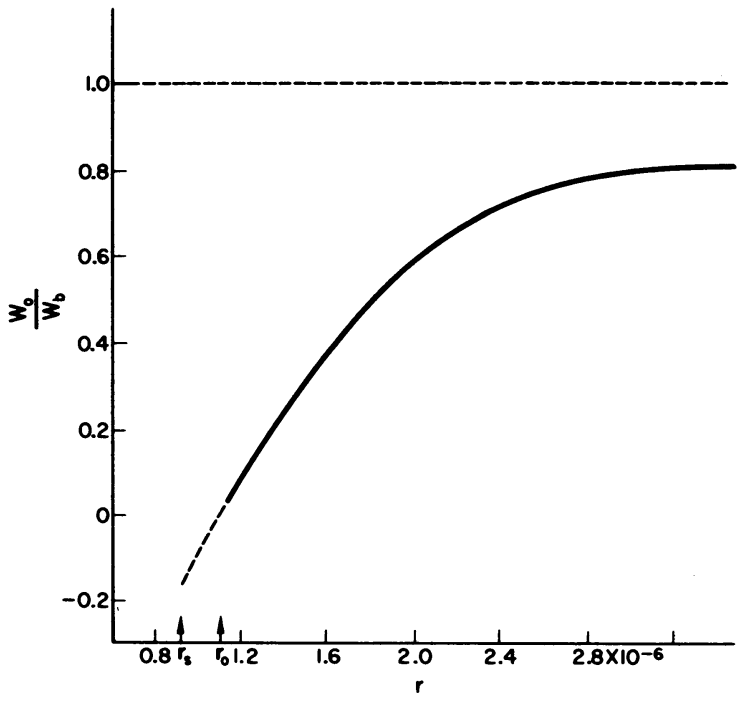

FIGURE 2

The ratio of motley to BCS condensation energies, $W_{0} / W_{b}$ at $T=0$ plotted against $r$. The dashed portion denotes the metastable region lying between $r_{s}$ and $r_{0}$.

\section{Equilibrium State at Finite Temperature}

In this section we generalize the preceding theory to describe the motley state at $T>0$. A novel feature of the excited state distribution function is that "two kinds" of excited particle compete for occupation. One behaves like a member of a thermally broken pair in that it makes no contribution to the neutralization of the pair deficiency $n_{1}$. The other behaves like an excited single and does contribute to neutralizing $n_{1}$. Since these "kinds" of excitation are otherwise indistinguishable, they combine in a nonseparable entropy function.

A svstem in the statistical ensemble is specified by stating, for each set of individual states $(k, s)+(-k,-s)$, whether exactly one is occupied by a single, by a member of a broken pair, or by neither of these, in which case it is available for pair occupancy. The distribution functions for these three cases are $l_{k}, g_{k}$, and $h_{k}$, respectively.

To determine the distribution functions, we require the free energy

$$
F=W-T S
$$

The quantum mechanical and thermal average of the Hamiltonian (2) is 


$$
\begin{aligned}
W & =\sum_{k}\left|\varepsilon_{k}\right|\left[l_{k}+2 g_{k}+2\left(1-l_{k}-2 g_{k}\right) h_{k}\left(\left|\varepsilon_{k}\right|\right)\right] \\
& -\cos \sigma \sum_{k, k^{\prime}} V_{k k^{\prime}}\left[h_{k}\left(1-h_{k}\right) h_{k} \cdot\left(1-h_{k}\right]^{1 / 2}\left(1-l_{k}-2 g_{k}\right)\left(1-l_{k}{ }^{\prime}-2 g_{k}^{\prime}\right)\right. \\
& +\frac{4 c e^{2}}{r^{4}}\left(\frac{1}{\sigma}-\frac{1}{2} r^{3} \sum_{k} l_{k}\right.
\end{aligned}
$$

The entropy is given by

$$
\begin{aligned}
-T S & =k T \sum_{k}\left[g_{k} \ln g_{k}+\left(1-g_{k}\right) \ln \left(1-g_{k}\right)\right. \\
& \left.+\left(l_{k}+g_{k}\right) \ln \left(l_{k}+g_{k}\right)+\left(1-l_{k}-g_{k}\right) \ln \left(1-l_{k}-g_{k}\right)\right]
\end{aligned}
$$

Minimizing $F$ with respect to $h_{k}$ as we did for the ground state, we obtain either $l_{k}+2 g_{k}=1$ or else the BCS-1ike set of equations:

$$
\begin{gathered}
h_{k}=\frac{1}{2}\left(1-\varepsilon_{k} / E_{k}\right), \quad\left[h_{k}\left(1-h_{k}\right)\right]^{1 / 2}=\frac{1}{2} \Delta / E_{k}, E_{k}^{2}=\varepsilon_{k^{2}+\Delta^{2},} \\
\Delta=V \cos \sigma \sum_{k^{\prime}}\left[h_{k^{\prime}}\left(1-h_{k^{\prime}}\right)\right]^{1 / 2}\left(1-l_{k^{\prime}}-2 g_{k^{\prime}}\right) .
\end{gathered}
$$

Again we have assumed a constant $V$ with a cutoff at $\omega$, the Debye frequency.

We further find

$$
\begin{gathered}
\frac{\partial F}{\partial l_{k}}=E_{k}-E_{2}+k T \ln \frac{l_{k}+g_{k}}{1-l_{k}-g_{k}}=0, \\
\frac{\partial F}{\partial g_{k}}=2 E_{k}+k T\left(\ln \frac{g_{k}}{1-g_{k}}+\ln \frac{l_{k}+g_{k}}{1-l_{k}-g_{k}}\right)=0 .
\end{gathered}
$$

Again we have used

$$
\sum_{k} l_{k}=2 N \varepsilon_{1}, \quad E_{2}=\frac{4 c e^{2}}{r}\left(\frac{1}{\sigma}-r^{3} N \varepsilon_{1}\right) \text {. }
$$


The solutions to (26) and (27) are

$$
g\left(E_{k}\right)=f\left(E_{k}+E_{2}\right), \quad l\left(E_{k}\right)=f\left(E_{k}-E_{2}\right)-f\left(E_{k}+E_{2}\right) .
$$

where $f$ is the Fermi function, $f(x)=[1+\exp (\beta x)]^{-1} ; \beta^{-1}=k t$. It is easy to see that $l+2 g \leqslant 1$, and also that $l$ and $g$ reduce to the appropriate values at $T=0$.

Minimizing $F$ with respect to $\sigma$ and using (28) in the resulting equation yields

$$
A(\sigma)=\sigma^{2} \tan \sigma \sec \sigma=\frac{2 V E_{2}}{r^{3} \Delta^{2}}
$$

A second expression relating $\sigma, \Delta$. and $E_{2}$ will come from equation (25), which we put into the form

$$
(N V \cos \sigma)^{-1}=\int_{0}^{\omega} \frac{d \varepsilon}{E}\left[1-f\left(E-E_{2}\right)-f\left(E+E_{2}\right)\right] .
$$

As before, the smallness of the ratio $E_{2} /\left(4 c e^{2} / r\right)$ allows us to change (28) to

$$
\varepsilon_{1}=\left(r^{3} N \sigma\right)^{-1}=\int_{0}^{\infty} d \varepsilon\left[f\left(E-E_{2}\right)-f\left(E+E_{2}\right)\right] \text {. }
$$

Extending the upper limit to infinity is permissible due to the rapid convergence for $E>k T$. We shall later show that (32) is valid, at least near the transition temperature, for all $r$. As before, $E_{2}=E_{1}$ at $T=0$. For $T$ not too close to zero, $E_{2}<\Delta$ and therefore $\varepsilon_{2}$ is not defined; this is not a defect.

We see by inspection of (30) - (32) that $\Delta$ can never reduce to zero at any temperature except in the BCS limit. We define $T_{c}$ as the maximum temperature for which a solution exists, and $T_{b}$ as the bulk maximum temperature.

We wish to solve (30) - (32) for the temperature dependence of the motley state parameters from 0 to $T_{c}$. To make headway we must drastically simplify the equations. Primarily we draw an analogy to a helpful solution of the BCS equation

$$
(N V)^{-1}=\int_{0}^{\omega} \frac{d \varepsilon}{E_{b}}\left[1-2 f\left(E_{b}\right)\right] \text {, }
$$

which is the bulk limit of equation (31). A close approximation to the general solution to (33), good regardless of coupling strength, is given by Swihart [7] based on a theory of Thouless [8]:

$$
\frac{\Delta_{b}(T)}{\Delta_{b}(0)}=\tanh \left[\frac{T_{b} \Delta_{b}(T)}{T \Delta_{b}(0)}\right]
$$

We are able to draw a rather complete analogy to this solution through the following argument. If we ignore the restriction imposed by equation (30), then we may define $T_{z}$ as the maximum temperature for which (31) has a solution for $\sigma=\sigma_{z} ; \Delta\left(T_{z}\right) \rightarrow 0$. Let us then define the general solution to (31) to be a Thouless-Swihart equation of the form 


$$
\frac{\eta(T)}{E_{1 z}(0)+\varepsilon_{1 z}}=\tanh \left[\frac{T_{z} \eta(T)}{T\left(E_{1 z}(0)+\varepsilon_{1 z}\right)}\right]
$$

In attempting to make sense out of (35), we are hindered by the fact that $\sigma$ changes with $T$. We overcome this difficulty by defining the subscript $z$ to mean that all quantities are given the values which they would have if $\sigma(0)$ and $\sigma\left(T_{z}\right)$ were set equal to the value $\sigma(T)$, where $T$ is the temperature at which the quantities $\Delta, \varepsilon_{1}, \sigma$ are being calculated. With this definition of $z$, we see that $\eta(T)=E_{2}(T)+\varepsilon_{2}(T)$ fits (35) at low enough $T$. We also see that $\eta(T)=\Delta(T)$ fits (35) at all $T$ if $\varepsilon_{1 z} \ll \Delta_{z}(0)$. We assume that

$$
\eta(T) \equiv \max \left[E_{2}(T)+\varepsilon_{2}(T), \Delta(T)\right]
$$

fits (35) at all $T$ for all samples.

We now recall equation (16), which says

$$
E_{1 z}(0)+\varepsilon_{1 z}=B\left(\sigma_{z}\right) .
$$

In weak-coupling $T_{z}$ and $\Delta_{z}(0)$ are fairly well related by

$$
\beta_{z}\left(E_{1 z}(0)+\varepsilon_{1 z}\right)=1.75 \text {. }
$$

Using equations (37) and (38), we convert (35) to

$$
1.75 k T=\frac{\eta(T)}{\tanh ^{-1}[\eta(T) / B(\sigma(T))]}
$$

The system of equations (30), (32), (36), and (39) contains the unknowns $\Delta(T), E_{2}(T), \sigma(T)$, plus the definition of $\eta$.

\section{Approximate Numerical Solution}

To obtain numerical solutions to the motley equations, we attempt to follow a plan similar to the one laid out for the $T=0$ case. We notice that equation (32) has two simple limiting solutions. (1) For $k T \ll \Delta(T), E_{2} \approx E_{1}$. In fact, $E_{2}$ at first slightly increases with $T$ due to the asymmetry in $N(E)$ around $E=E_{1}$. For $T$ a bit larger, the asymmetry is reversed because of the energy gap, and $E_{2}$ begins to decrease below $\Delta$. This occurs when $E_{1}(T)-k T \approx \Delta(T)$. (2) For $k T \gg \Delta(T),\left(r^{3} N \sigma\right)^{-1} \approx E_{2}$. A picture of the Fermi functions gives this result by inspection. In between (1) and (2) a rough interpolation can be made.

Once the simplification (1) or (2) has been made, we solve for $\sigma(T)$ and $\Delta(T)$ as we solved before for $\sigma(0)$ and $\Delta(0)$. There are two reasons for isolating $T$ on the left side of (39). One is that it is then simpler to find $T_{c}$. Another arises when it turns out that $\sigma$ and, especially, $\Delta$ vary rapidly near $T_{c}$ and furthermore are double-valued over a wide range of $T$. An example of this behavior for the sample value $r=160 \AA$ is displayed in Fig. 3. The double-valuedness means that the free energy has two minima. The absolute minimum lies in the solid lines. The secondary minimum does not persist down to $T=0$. 


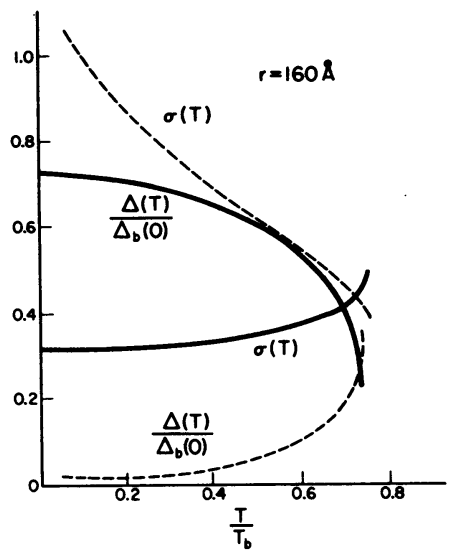

FIGURE 3

The motley parameters, $\sigma(T)$ and $\Delta(T) / \Delta_{b}(0)$, vs. $T / T_{b}$ for $r=160 \AA$. The absolute minimum of the free energy lies in the solid curves. The dashed curves denote unphysical solutions.

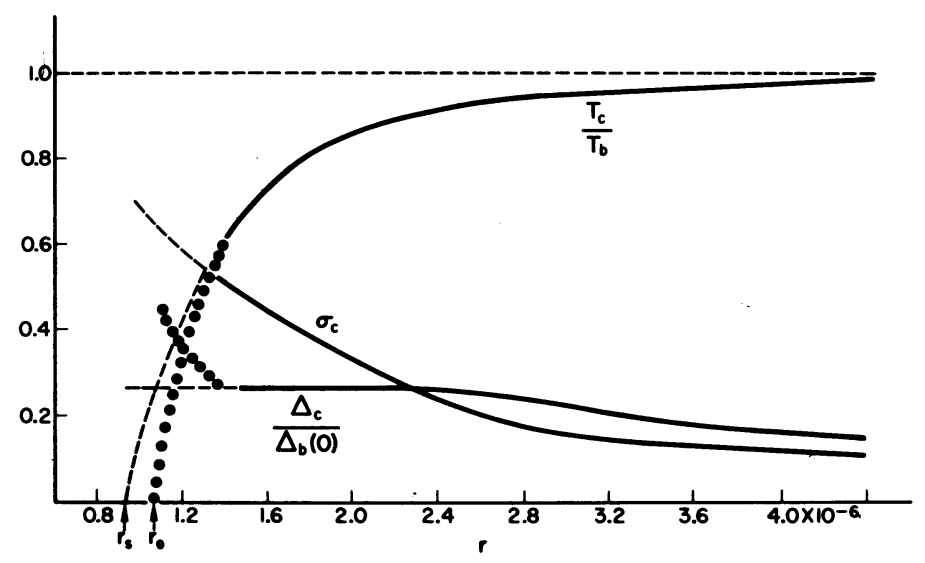

FIGURE 4

The motley parameters, $T_{c} / T_{b}, \sigma_{c}$, and $\Delta_{c} / \Delta_{b}(0)$, vs. $r$. Subscripts $c$ and $b$ refer to the critical temperature and to the bulk material, respectively. The dashed portions lie in the region of instability and begin at $r_{s}$. The dotted portions are the values at the "transition point" defined at the end of the next section and begin at $r_{0}$. 
The critical temperature $T_{c}$ occurs at the value of $T$ where the solid and dashed curves intersect, 1.e. at the point where the free energy has a single minimum. To the right of that point, i.e. at higher values of $T$, there exists no solution to the equations. However, for purposes of illustration, we have extended the curves beyond $T_{c}$ to show the accuracy in the procedure of locating the critical parameters. We see from Fig. 3 that approximations used in setting up and solving the equations will produce a relatively large error in the value of $\Delta_{c}$ due to the steepness of the curves for $\Delta_{c}$ near $T_{c}$. A smaller error will result in the value of $\sigma_{c}$ due to the gentler slope. Most important, the error in locating $T_{c}$ will be very slight because it is not as sensitive to the approximations.

In Fig. 4 we have plotted vs. $r$ the values of these critical parameters. In Fig. 5 we have shown rough curves of $\Delta(T)$ vs. $T$ for a number of samples. The initial slope at $T=0$ of $\Delta(T)$ is

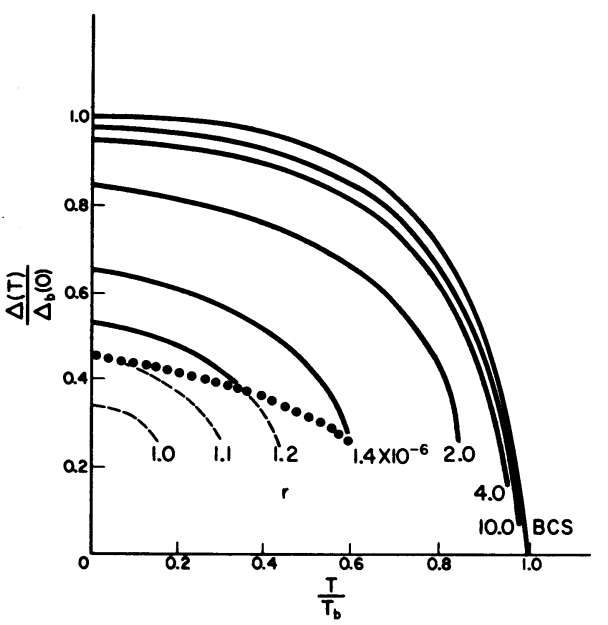

FIGURE 5

\begin{abstract}
Rough curves of $\Delta(T) / \Delta_{b}(0)$ vs. $T / T_{b}$ for several values of $r$. The dashed portions lie in the region of instability. The dotted curve represents the locus of values at the "transition point" defined at the end of the next section.
\end{abstract}

zero. This is also true of $\sigma(T)$ and $E_{2}(T)$. From $T_{c}$ or $\Delta_{c}$ or the curve $\Delta(T)$, we decide that again $r \approx 600 \AA$ is close to being "bulk" material.

Limiting expressions for large $r$ are obtained by maximizing $T$ in equation (39) subject to the constraint (30) and the approximation of case (2). These expressions are $\sigma_{c} r=4.3 \times 10^{-7}$, $T_{c}=\left(1-2.2 \sigma_{c}{ }^{2}\right) T_{b}, \Delta_{c}=1.5 \sigma_{c} \Delta_{b}(0), E_{2 c}=1.2 \sigma_{c} \Delta_{c}$. The numerical coefficients depend on the values of $N$ and $V$. With our representative values, $\sigma_{c} \approx \sigma_{0}$ and, therefore, $\Delta_{c} \approx 1.5 \sigma_{c} \Delta_{0}$. That is, $\sigma$ is unchanged and $\Delta$ decreases between 0 and $T_{c}$. We can show that near $T_{c}$, just as at $T=0, \sigma^{-1} \gg E_{2} /\left(4 c e^{2 / r}\right)$ for all $r$ and hence equation (32) is valid for all $r$. We believe this is true at all $T$. 


\section{Free Energy and Specific Heat}

We saw earlier that in the tiniest samples the motley state is unstable relative to the normal state at $T=0$. We inquire about this situation at $T>0$, and we also ask for the specific heat and for the latent heat of the first order transition at $T_{c}$. These quantities are obtained by substituting the expressions derived for the distribution functions into equations (21) - (23).

Since we follow the well known procedure of BCS step by step, we shall write down mainly the results. In the weak-coupling regime, we find for the free energy difference between motley and normal states,

$$
\begin{aligned}
\frac{F_{m}-F_{n}}{W_{b}} & =\frac{\Delta^{2}}{\Delta_{b}(0)^{2}}+\frac{2}{\Delta_{b}(0)^{2}} \int_{0}^{\infty}\left[\frac{2 \varepsilon^{2}+\Delta^{2}}{E}\left\{f\left(E-E_{2}\right)+f\left(E+E_{2}\right)\right\}\right. \\
& \left.-2 E_{2}\left\{f\left(E-E_{2}\right)-f\left(E+E_{2}\right)\right\}\right] d \varepsilon \\
& -\frac{2}{3} \pi^{2} \frac{(k T)^{2}}{\Delta_{b}(0)^{2}}-\frac{2 E_{2}^{2} / \Delta_{b}(0)^{2}}{\left(4 c e^{2} / r\right) r^{3}},
\end{aligned}
$$

where the normal state free energy is

$$
F_{n}=-\frac{1}{3} \pi^{2} N(k T)^{2} \text {. }
$$

and $W_{b}=-1 / 2 N \Delta_{b}(0)^{2}$ As before, the last term in (40) is negligible for all $r$.

We are unable to make a satisfactory plot of (40) for a small value of $r$. Even if we could, it would not represent a direct experimental quantity. In the BCS case, the connection with experiment comes from $H_{b}^{2} / 8 \pi=F_{n}-F_{s}$, where $H_{b}$ is the bulk critical field. The critical field in the case of a small sample depends on the sample size as well as on $F_{n}-F_{m}$. Furthermore, we have neglected Pauli paramagnetism, which becomes important for high critical fields.

The electronic specific heat in the motley state is given by

$$
C_{e m}=T \frac{d S}{d T}=-\beta \frac{d S}{d \beta} .
$$

Again following BCS, we find, in analogy with their equation (3.46),

$$
\begin{gathered}
C_{e m}=2 k \beta^{2} \sum_{k>k_{F}}\left\{f ( E - E _ { 2 } ) [ 1 - f ( E - E _ { 2 } ) ] \left[\left(E-E_{2}\right)^{2}+\beta\left(E-E_{2}\right)\right.\right. \\
\left.\left.\quad \times\left(\frac{1}{2 E} \frac{d \Delta^{2}}{d \beta}-\frac{d E_{2}}{d \beta}\right)\right]+\left(-E_{2} \rightarrow+E_{2}\right)\right\} .
\end{gathered}
$$


For region (1), defined at the opening of the preceding section,

$$
C_{e m} \approx 2 k \beta^{2} N \int_{\Delta \varepsilon}^{\infty} \frac{E}{\varepsilon}\left(E-E_{1}\right)^{2} f\left(E-E_{1}\right)\left[1-f\left(E-E_{1}\right)\right] d E .
$$

Compare this to the normal state:

$$
C_{e n} \approx 4 k \beta^{2} N \int_{0}^{\infty} \varepsilon^{2} f(\varepsilon)[1-f(\varepsilon)] d \varepsilon
$$

The asymmetry in $N(E)$ is a secondary effect. The primary effect is the relative magnitudes of $N\left(E_{1}\right)$ and $N$. That is, the specific heat ratio is

$$
C_{e m} / C_{e n} \approx \frac{1}{2} E_{1} / \varepsilon .
$$

for very low $T$. As $T$ rises, $C_{e m} / C_{e n}$ falls quickly below one until, in region (2), $C_{e m} / C_{e n}$ rises to a value greater than one. It is suitable to mention here that the same approximation applied to equation (40) would show an initial increase in the free energy advantage of the motley state, in direct contrast to the BCS case.

In Fig. 6 we have plotted $C_{e m} / C_{e n}$ vs. $r$ for two different low temperatures. (This and the remaining two figures are only very roughly accurate.) The graph shows that a value $T=0.1 T_{b}$

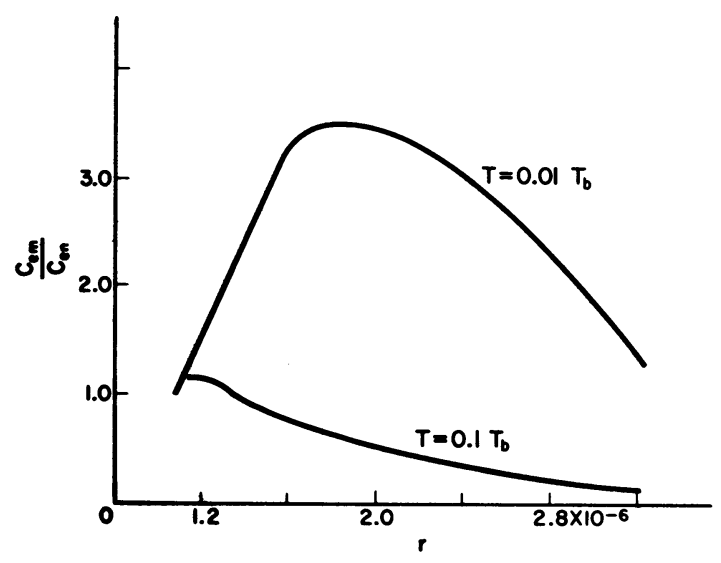

FIGURE 6

The (motley-to-normal) specific heat ratio, $C_{e m} / C_{e n}$, vs. $r$ for two different low temperatures, $T=0.01 T_{b}$ and $0.1 T_{b}$.

is not low enough to give $C_{e m} / C_{e n}>1$. Only for $T \sim 0.01 T_{b}$ will a sample consisting of beads from 100 to $300 \AA$ give a ratio rising substantially above one. This is a very low temperature, generally $0.01-0.05^{\circ} \mathrm{K}$.

In Fig. 7 we depict the temperature variation of $C_{e n}$, the BCS $C_{e s}$, and the present $C_{e m}(r)$ 
for two small values of $r$, namely $150 \AA$ and $250 \AA$. All the curves are normalized to $\gamma T_{b}$, the value of $C_{e n}\left(T_{b}\right)$. The spikes at $T_{c}$ in the motley case represent the latent heat. The difference in area between a motley curve and the normal line from 0 to $T_{c}$, plus the value of the spike, together equal the condensation energy, $-W_{0}$, at $T=0$. $W_{0}^{\prime}$ was plotted in Fig. $2 ; W_{b}=0.23 \gamma T_{b}^{2}$ Anticipating the material in the next paragraph, we may state that the latent heat is a small fraction of the total enthalpy at $T_{c}, 0.015$ for $r=150 \AA, 0.007$ for $r=250 \AA$.

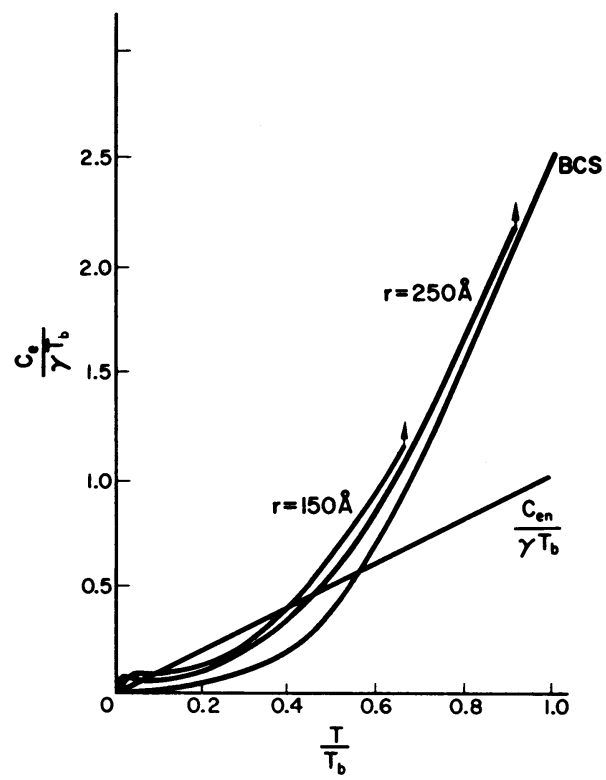

FIGURE 7

Specific heats, (normal) $C_{e n}$, (BCS) $C_{e s}$, and (motley) $C_{e m}(r)$ for $r=150 \AA$ and $250 \AA$, plotted vs. $T / T_{b}$. The spikes represent latent heats.

In Fig. 8 the latent heat, $W_{c}$, is plotted as a function of $T_{c}$, whose $r$-variation is indicated. In this rough graph, only for $r>r_{c} \approx 140 \AA$ is there a positive latent heat. For $r<r_{c}$ the motley state is unstable at $T_{c}$. Therefore, while $T_{c}$ remains the highest value of $T$ for whic a mathematical solution exists, the physical "transition temperature" at which the normalmotley transition occurs falls below $T_{c}$. This temperature, symbolized as $T_{t}$, corresponds to the point where the normal and motley free energies are equal; i.e. $F_{n}\left(T_{t}\right)=F_{m}\left(T_{t}\right)$. A particular value $T_{t}$ belongs to a particular sample size $r$, which we may call $r_{t}$. Calculation finds that $r_{t}=r_{0} \approx 110 \AA$ at $T_{t}=0$; then $r_{t}$ decreases slightly as $T_{t}$ increases from zero; after that $r_{t}$ turns around and increases to $r_{c} \approx 140 \AA$ at that transition temperature such that $T_{t}=T_{c}$, where $T_{c}$ is once again the solution to the motley equations. We find that this occurs at $T_{t}=$ $T_{c}=0.57 T_{b}$, above which the usual solution applies and the latent heat is positive.

The region of instability of the motley state ("negative latent heat") is indicated by dashed lines in Fig. 8 and also in Figs. 4 and 5. Moreover, the dotted curves in Figs. 4 and 5 show roughly the behavior of $T_{t}$ and $\Delta_{t}$ as they vary with $r$. 


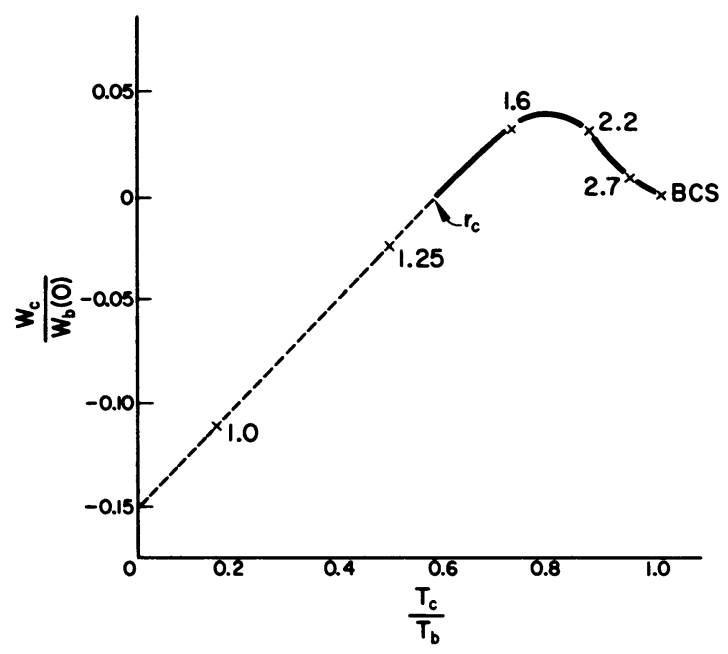

FIGURE 8

\begin{abstract}
Latent heat, $W_{c}$ vs. $T_{c}$ and also vs. $r . W_{b}(0)$ is the bulk condensation energy and $T_{b}$ is the bulk critical temperature. $r_{c}$ labels the value of bead size below which the normal state is stable below $T_{c}$ and above the "transition temperature" $T_{t}$ discussed in the text.
\end{abstract}

It is amusing to realize that at $T_{t}$ the transition mimics second order transitions by having zero latent heat. However, it possesses a basic property of first order transitions, which is a discontinuity in order parameter, $\Delta_{t} \neq 0$.

\title{
8. Other Properties, Particularly the Knight Shift
}

We have considered the motley state in the absence of a magnetic field. We have seen that a slight rise in temperature above zero favors the motley state due to the enhanced density of states of the singles. For the same reason a small field $H$ favors the motley state at $T \approx 0$, due to spin paramagnetism. If the field is small enough $\left(H \ll H_{c}\right)$ that the alteration in pairing and related complications are small, then the paramagnetic effect dominates. The criterion for $H$ analogous to region (1) for $T$ (see the paragraph following equation (39)) is $E_{1}-\mu H \approx \Delta$; $\mu$ is the Bohr magneton. By analogy with Fig. 6, we may state that for $\mu H \approx 0.01 k T_{b}$, the paramagnetic susceptibility for a sample of beads from $100 \AA$ to $300 \AA$ is higher in the motley than in the normal state, $X_{m}>X_{n}$. For $\Delta_{b}=10^{-3} \mathrm{v}, H \approx 1 \mathrm{~kg}$. This prediction is in complete contrast to the BCS theory, which predicts $X_{s} / X_{n} \rightarrow 0$ as $T \rightarrow 0$.

Knight shift experiments $[9,10]$ which measure $X$, have for years been performed on samples well suited to us: beads of dimension $100-400 \AA$. Extrapolation from $T$ above $0.2 T_{b}$ indicates that $X_{s} / X_{n} \approx 2 / 3$ at $T=0$. Thus there is experimental disagreement with both this paper and with BCS. Several theoretical papers [11] have reconciled BCS with experiment, primarily by invoking spin-orbit coupling to disrupt the perfect singlet pairing. 
Apparently much more serious is the fact that other measured properties of these samples, in addition to the Knight shift, systematically contradict the predictions of this paper. For example, $T_{c}$ was determined to be practically equal to $T_{b}$, whereas we predict a fairly strong depression of $T_{c}$ due to the electrostatic free energy. We plan to show, however, that the experimental conditions under which these measurements were taken were in violation of a single condition critical for the detection of the electrostatic effect. The argument is part of the following general discussion [12].

It was shown in an earlier section that the size of the domain across which a charge fluctuation exists is the size of the sample. This agrees with the well-known fact that in a metal the long-range part of the Coulomb interaction is screened out even in the normal state. Thus the actual charge of a quasi-particle always appears on the surface of the sample. Each electro is essentially coupled to a capacitor of magnitude $r$, where $r$ is the sample size [13].

We can go a crucial step further than this. Pursuing the idea of screening of the Coulomb interaction, we see that the considerations of this paper would not be true for beads in actual electrical contact; the impedance has to be no less than the capacitive coupling. This means that the distance between neighboring beads must be (much) greater than the size of a single bead. For the powdered samples in the Knight shift experiments [9], this is exactly the critica. condition which was violated. In fact the average distance between neighboring beads was somewhat smaller than the size of a typical bead. For this reason we make the claim that the presen theory has not yet been put to experimental test.

If appropriate samples can be made, then the predictions of this article can be tested. Thos most apt to be measured are the depressed critical temperature and enhanced density of states at very low temperature $\left(0.01-0.05^{\circ} \mathrm{K}\right)$.

In summary, the properties which reflect the density of states are Knight shift at fields such that $E_{1}-\mu H \approx \Delta$, specific heat, spin-lattice relaxation rate $\left(\tau^{-1} \propto N^{2}\right)$, and microwave absorption at a frequency $\omega$ such that $E_{1}-\hbar \omega>\Delta$. In the last two properties the coherence factors of case II of BCS play a role; in fact they act favorably since they slightly increase $N_{m} / N_{n}$.

The Cryogenics Group at the University of Connecticut is looking into the matter of preparing appropriate samples. Standard methods for making powdered samples are not applicable here because they do not separate the beads to distances larger than the size of the beads.

\section{Concluding Remarks, Including Self-Criticism}

We now turn to questions of the accuracy of the motley equations and of their solution. First of all, we have ignored the dynamics of the charge and phase. A proper account would bring in other energy terms, e.g. kinetic energy of the currents. Secondly, we have not accounted for several effects of the smallness of the beads: the parameters $N, V$, and $\omega$ will not have their bulk values; the Bloch levels $\varepsilon_{k}$ are quantized; perhaps there should be a surface term in the Hamiltonian. Thirdly, the variational wave function is an approximate solution to the reduced Hamiltonian, shown by BCS in their appendix A to be an exact solution in the limit $r \rightarrow \infty$. Our limit, $r$ small, is undoubtedly the region of greatest inaccuracy. Fourthly, S. Engelsberg (private communication) has cautioned us that the interaction $H_{e}$ would tend to renormalize the $\varepsilon_{k}$ vs. $k$, thus changing $N(\varepsilon)$ and $N(E)$, if it were treated in a dynamical way. Finally, Anderson (private communication) has pointed out an additional effect which smallness of the sample. 
brings about: the smearing out of the transition singularity of whatever kind for the reason that $Z=\operatorname{Tr}\left[e^{-H / k T]}\right.$ can have no mathematical singularities except in the limit as $n \rightarrow \infty$, in principle [14]. ( $n$ is the total number of particles.)

In line with certain of these considerations, we point out that a previous estimate of the minimum size of a superconductor, based upon the separation of quantized Bloch energies being roughly equal to the bulk energy gap, was made by Anderson [15]. It would be $r \approx 50 \AA$ for our values of $N$ and $\Delta_{b}$, which is smaller than the estimate of $110 \AA$ according to the present theory. This would seem to indicate that quantization of the Bloch levels is not critical to the motley state. We might guess that, due to the form of $Z$, the smearing of the transition singularity is of the same relative importance as the quantization of levels. All in all, however, we have no real criteria with which to rule out any of the criticisms in this section.

It is important to realize that, whether or not the electrostatic effect actually operates in a tiny superconductor, ultimately some size effect quenches superconductivity. Most (or all) of us would say instinctively that a bead consisting of ten atoms could not be a superconductor (is it even a metal?) simply because there are not enough electrons around to do the job. This is essentially Anderson's criterion [15]. Thus there must be a minimum size to a sample. Experimentalists will sooner or later measure this size. One will have to be extremely cautious when attributing the quenching effect to one particular mechanism. Regarding the electrostatic effect, the strong dependence on the distance between beads may allow unambiguous interpretation. It is hard to conceive of another mechanism so sensitive to the isolation of the beads.

Two speculative ideas are forwarded contingent upon the detection of the motley state. First, the organic superconductors discussed by Little [16] are of a size to be affected by the considerations of this paper. The existence of those essentially one-dimensional superconductors has been questioned by Ferrell [17], who pointed out an instability with respect to the formation of compressional modes, which themselves destroy superconductivity in one dimension. Second, other types of condensed system might be shown to obey theories similar to this one; for example, superfluid, ferromagnetic, or the tiniest condensed system of all, the atomic nucleus. Naturally the form of interaction is different in each.

According to recent theoretical work [18], the size dependence of thermal fluctuations of the superconducting order parameter would be sufficient to smear the phase transition by an amount $\delta T \sim T_{c}$ for sample dimension $\sim 100 \AA$. Such an effect would dominate the electrostatic effect discussed in the present paper. However, experimentally it is found that the transition width is not much larger than in bulk specimens, of the order of millidegrees [9, 10]. Note that electrical isolation of the $100 \AA$ beads is not a requirement for thermal fluctuations. Therefore, apparently we need not consider thermal fluctuations.

\section{Acknowledgements}

We are extremely grateful to Drs. B. Schwartz and T. Tsuneto for imparting to us some of their fine physical insight into the mechanism of the Josephson effect and of the present model. We are particularly indebted to Dr. P.W. Anderson for his advice and encouragement. We gratefully acknowledge Professor C.A. Reynolds for a critical reading of the manuscript. 


\section{References}

1. B.D. JOSEPHSON, Phys. Letters 1, 251 (1962).

2. P.W. ANDERSON, Lecture entitled Weak Superconductivity: The Josephson Tunneling Effect delivered at the Spring School of Physics at Ravello, Italy, Benjamin, New York (1963).

3. J.BARDEEN, L.N. COOPER and J.R. SCHRIEFFER, Phys. Rev. 108, 1175 (1957).

4. A discussion of zero-point fluctuations and their relation to broken symmetries is given by P.W. Anderson, Concepts in Solids, p. 175. Benjamin, New York (1963).

5. A complete set of states requires introduction of "excited-pairs" generated by $\left[e^{i} \varphi^{\prime}\left(1-h_{k}\right)^{1 / 2} b_{k}{ }^{+}-h_{k}^{1 / 2}\right]$. The BCS procedure is to lump single-particle and excitedpair occupancies into a distribution function giving the overall probability of excited state occupancy. We use the same procedure. The resulting function $l_{k}$ belongs with an operator $a_{k}{ }^{+}$which creates any excitation with wave-vector $k$. To avoid implying that excitations are present in the ground state, we will substitute the term "singles".

In obcaining equation (5), we have replaced the average of a function of $\left|\phi_{-}-\varphi^{\prime}\right|$ by the function of the average of $\left|\varphi-\phi^{\prime}\right|$. which is $\sigma$. This approximation, like the Hartree assumption, is based on the presumed strong peaking of the weights of states with different phase spreads about $\sigma$. The approximation worsens as $\sigma$ increases.

7. J.C. SWIHART, IBM Jl Res. Dev. 6, 14 (1962).

8. D.J. THOULESS, Phys. Rev. 117, 1256 (1960).

9. F. REIF, Phys, Rev. 106, 208 (1957); G.M. ANDROES and W.D. KNIGHT, Phys. Rev. Let ters 2, 386 (1959); Phys. Rev. 121, 779 (1961).

10. In addition, an early paper by Shoenberg reported on the susceptibility of emulsive samples of sizes just on the verge of being useful to us: namely, a few hundred angstroms.

(D. Shoenberg, Proc. R. Soc. A175, 49 (1940). ) The electrostatic effect was not detected. The discussion in the text concerning Knight shift samples applies to these samples as well.

11. R. A. FERRELL, Phys. Rev. Letters 3, 262 (1959); P.C. MARTIN and L.P. KADANOFF, Phys. Rev. Letters 3, 322 (1959); J.R. SCHRIEFFER, Phys. Rev. Letters 3, 323 (1959); P. W. ANDERSON, Phys. Rev. Letters 3, 325 (1959); L.N. COOPER, Phys. Rev. Letters 8, 367 (1962); A.A. ABRIKOSOV and L.P. GORKOV, Soviet. Phys. JETP 12, 337 (1961); also JETP 15, 752 (1962).

12. This argument is due to P.W. ANDERSON, private communication. Also refer to Ref. 4 , p.109.

13. A dimensionless constant of order one should multiply $r$ as it does in $H_{e}$ in equation (2), but it is unimportant in this discussion.

14. See, for Instance, KERSON HUANG, Statistical Mechanics, Chapter 15. John Miley, New York (1963).

15. P.W. ANDERSON, J. Physics Chem. Solids 11, 26 (1959). 
16. W. A. LITTLE, Phys. Rev. 134, A1416 (1964).

17. R. A. FERRELL, Phys. Rev. Letters 13, 330 (1964).

18. G. LUCAS and M.J. STEPHEN, Phys. Rev. 154, 349 (1967). 